\title{
Correction: Xylem Functional Traits as Indicators of Health in Mediterranean Forests
}

\author{
Marco Borghetti ${ }^{1} \cdot$ Tiziana Gentilesca $^{1} \cdot$ Michele Colangelo $^{2} \cdot$ Francesco Ripullone $^{1} \cdot$ Angelo Rita $^{1}$ \\ Published online: 8 August 2020 \\ (C) Springer Nature Switzerland AG 2020
}

\section{Correction: Current Forestry Reports \\ https://doi.org/10.1007/s40725-020-00124-5}

The original version of this article unfortunately contained a mistake. The figures previously used are the old version and some symbols in the figure captions were displayed incorrectly. Final versions of figures with their captions are provided here.

The original article has been corrected.

Publisher's Note Springer Nature remains neutral with regard to jurisdictional claims in published maps and institutional affiliations.

The online version of the original article can be found at https://oi.org/ $10.1007 / \mathrm{s} 40725-020-00124-5$

Marco Borghetti

marco.borghetti@unibas.it

Tiziana Gentilesca

tiziana.gentilesca@gmail.com

Michele Colangelo

michele.colangelo3@gmail.com

Francesco Ripullone

francesco.ripullone@unibas.it

Angelo Rita

angelo.rita@unibas.it

1 Scuola di Scienze Agrarie, Forestali, Alimentari ed Ambientali (SAFE), Università della Basilicata, viale dell'Ateneo Lucano 10, 85100 Potenza, Italy

2 Instituto Pirenaico de Ecología (IPE-CSIC), Avda. Montañana 1005, Apdo. 202, 50192 Zaragoza, Spain 


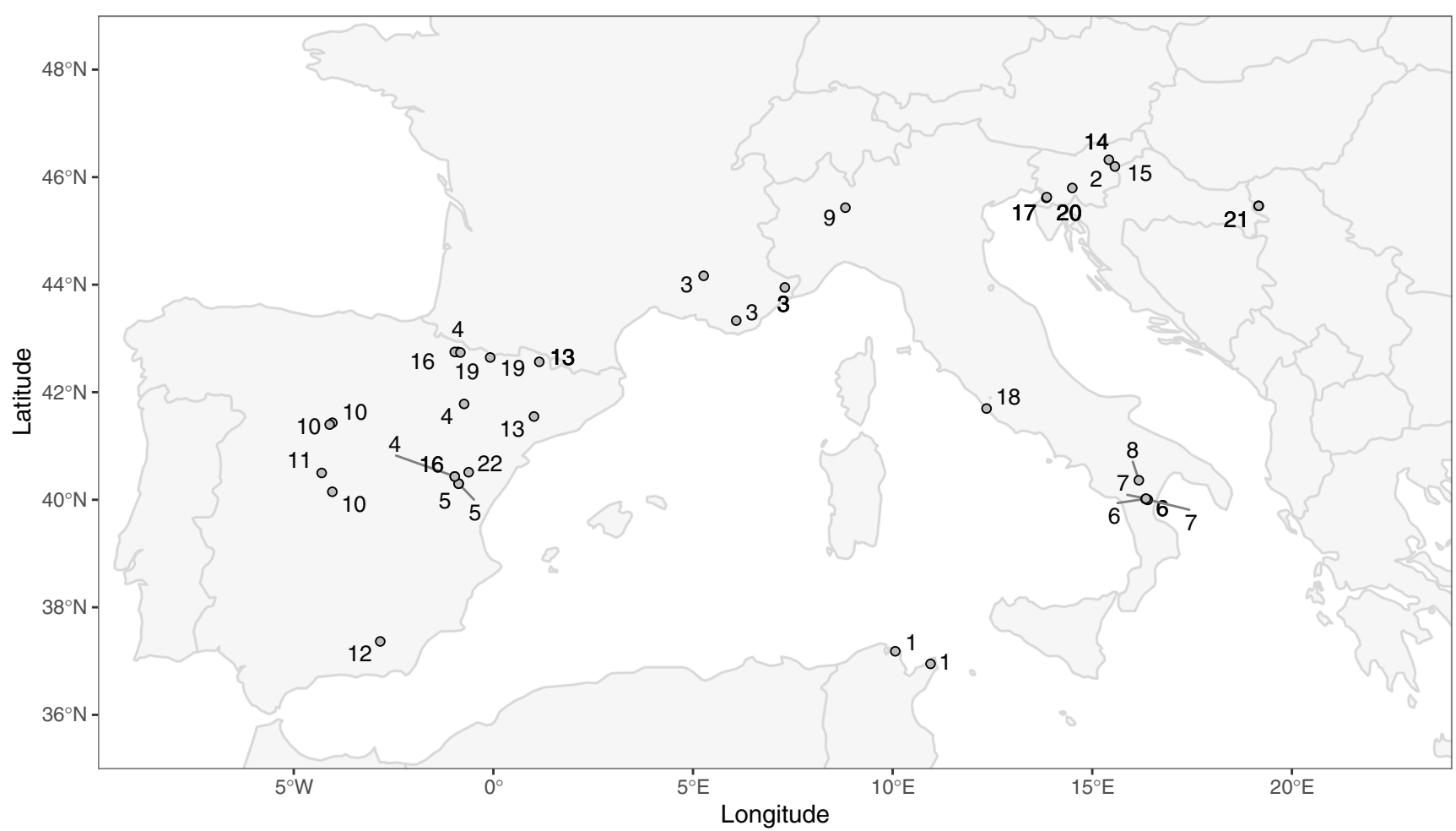

Fig. 1 Geographic distribution of selected case studies with investigated tree species. Numbers in open circles refer to the articles listed in Table 1, from which tree-ring width, basal area increment, and xylem trait chronologies were compiled for the present meta-analysis 

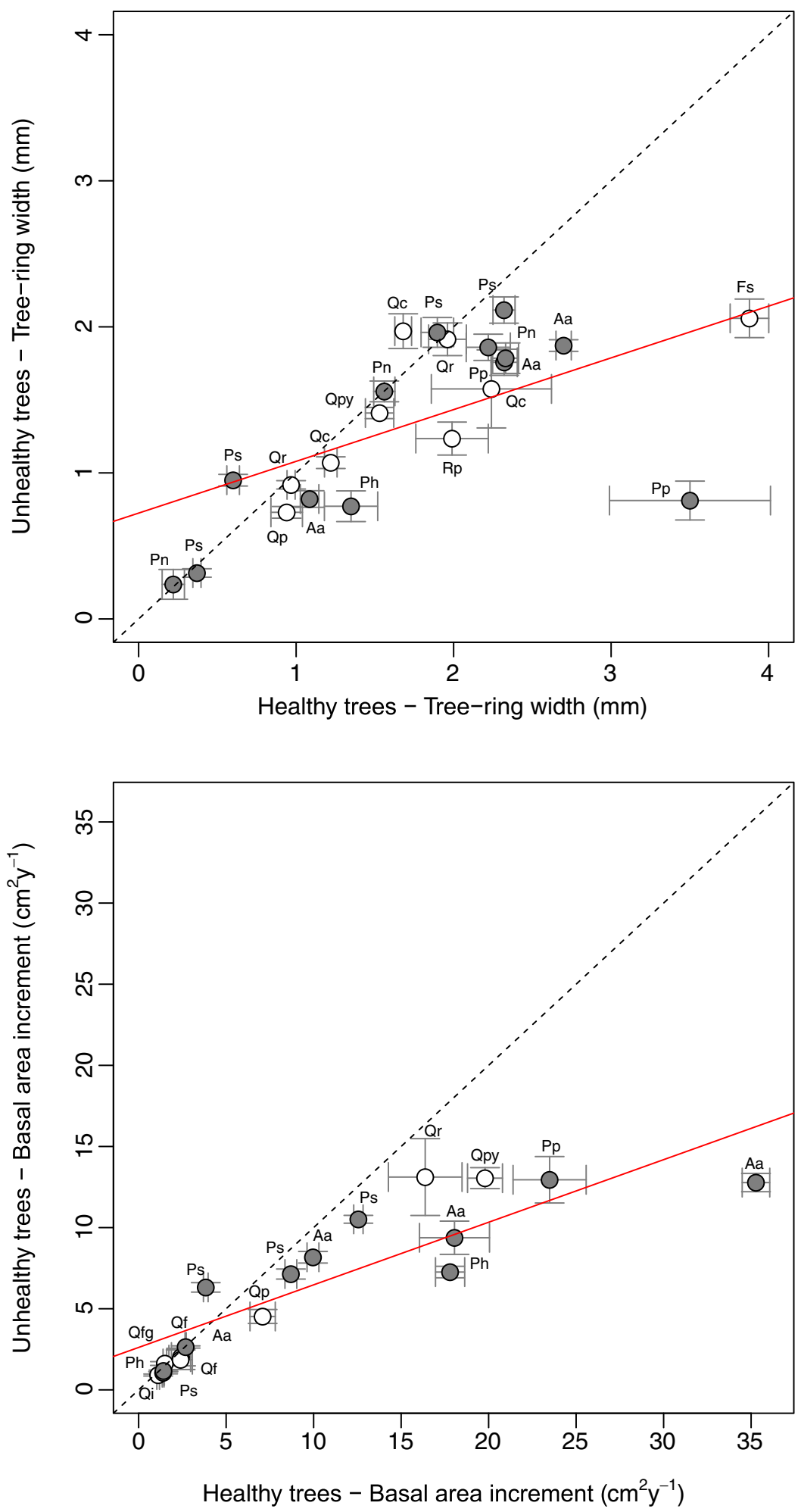

Fig. 2 Results of meta-analyses of tree-ring width (TRW, upper panel) and basal area increment (BAI, lower panel) values compiled from study cases listed in Table 1. The relationship between $\mathrm{H}$-trees and $\mathrm{U}$-trees is represented in both panels. Circles and bars represent mean values and standard deviations for each investigated tree species, respectively. The dashed lines show 1:1 relationship. The solid red lines are the regression predictions obtained from the applications of a linear mixed-effect model in which tree species were considered as random terms (TRW: $Y$ - intercept $=0.72$, slope $=0.35, R^{2}$ marginal $=0.36 ; R^{2}$ conditional $=0.76$, $p<0.01$; BAI: $Y$-intercept $=2.61$, slope $=0.38, R^{2}$ marginal $=0.78 ; R^{2}$ conditional $=0.98, p<0.001)$. Gray and white circles are conifer and hardwoods, respectively. Ps, Pinus sylvestris; Pn, Pinus nigra; Ph, Pinus halepensis; Pp, Pinus pinaster; Aa, Abies alba; Qr, Quercus robur; Qc, Quercus cerris; Qp, Quercus pubescens; Qpy, Quercus pyrenaica; Qf, Quercus frainetto; Qfg, Quercus faginea; Qi, Quercus ilex; Fs, Fagus sylvatica; Rp, Robinia pseudoacacia 
Study

Earlywood

Colangelo et al (2017a) Quercus frainetto

Colangelo et al (2017b) Quercus frainetto

Colangelo et al (2018) Quercus robur

Jevsenac \& Levanic (2015) Quercus robur

*Levanic et al (2011) Quercus robur

Pellizzari et al (2016) Abies alba

Pellizzari et al (2016) Pinus sylvestris

Petrucco et al (2017) Pinus nigra

**Savi et al (2019) Pinus nigra

Random effects model

Heterogeneity: $I^{2}=95 \%, \tau^{2}=0.0174, p<0.01$

Latewood

Colangelo et al (2017a) Quercus frainetto

Colangelo et al (2017b) Quercus frainetto

Random effects model

Heterogeneity: $I^{2}=0 \%, \tau^{2}=0.0012, p=0.32$

Random effects model

Prediction interval

Heterogeneity: $I^{2}=94 \%, \tau^{2}=0.0141, p<0.01$

Residual heterogeneity: $I^{2}=95 \%, p<0.01$
Conduit area RMD

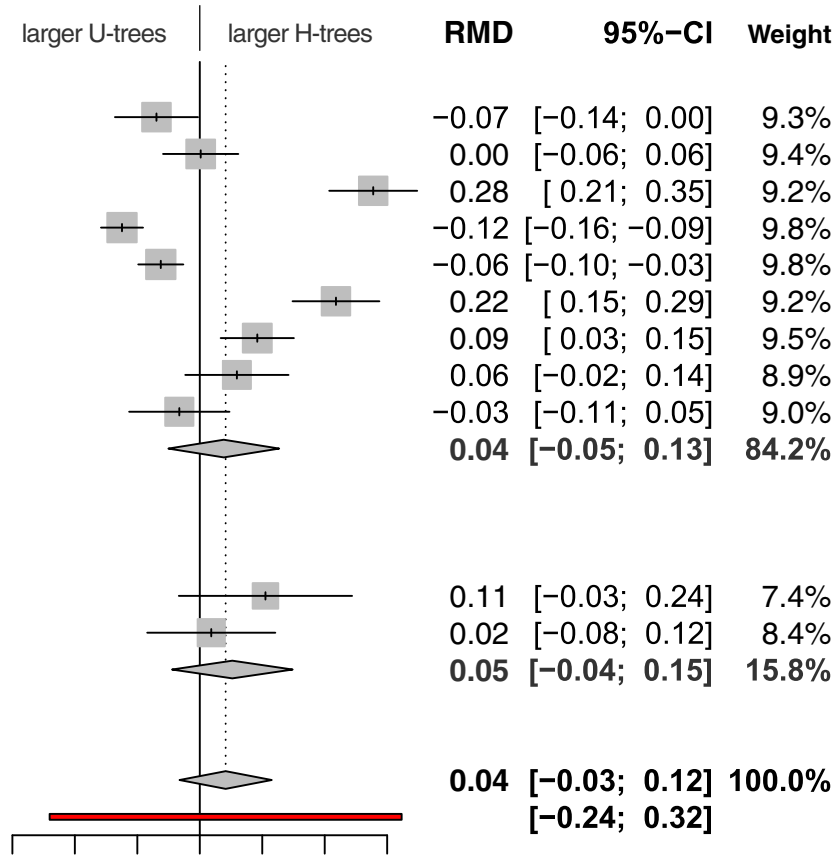

$\begin{array}{lllll}-0.3-0.2-0.1 & 0 & 0.1 & 0.2 & 0.3\end{array}$
Fig. 3 Meta-analysis results for xylem conduit areas (average lumen size) assessed before the drought-induced tree decline on wood chronologies from study cases listed in Table 1. Results for earlywood conduits and latewood conduits are shown in the upper panel and lower panel, respectively. From left to right, columns report authors, year of publication, and investigated tree species for selected cases. Graphical representation of the relative mean difference $(\mathrm{RMD}=\mathrm{MD} / \mathrm{x}$, where $\mathrm{MD}$ is the mean difference of samples and $\mathrm{x}$ is the sample mean) between $\mathrm{H}$-trees (trees that did not show symptoms of decline) and $\mathrm{U}$ trees (trees that showed symptoms of decline or were dying); error bars indicate $95 \%$ confidence intervals (CIs), the vertical dashed line represents the no effect line. The diamonds represent summary RMDs, such that the lateral diamond tips show associated CIs; $I^{2}$ is the variability percentage independent of sampling error; $\tau^{2}$ is the between-study variance. For each study, the weight is computed as the inverse of within-studies squared standard error plus the between-studies variance, $\tau^{2}$. The red line shows the range in which we can expect the effects of future studies to fall based on the current meta-analysis. *, hydraulic diameter; $* *$, radial lumen diameter 

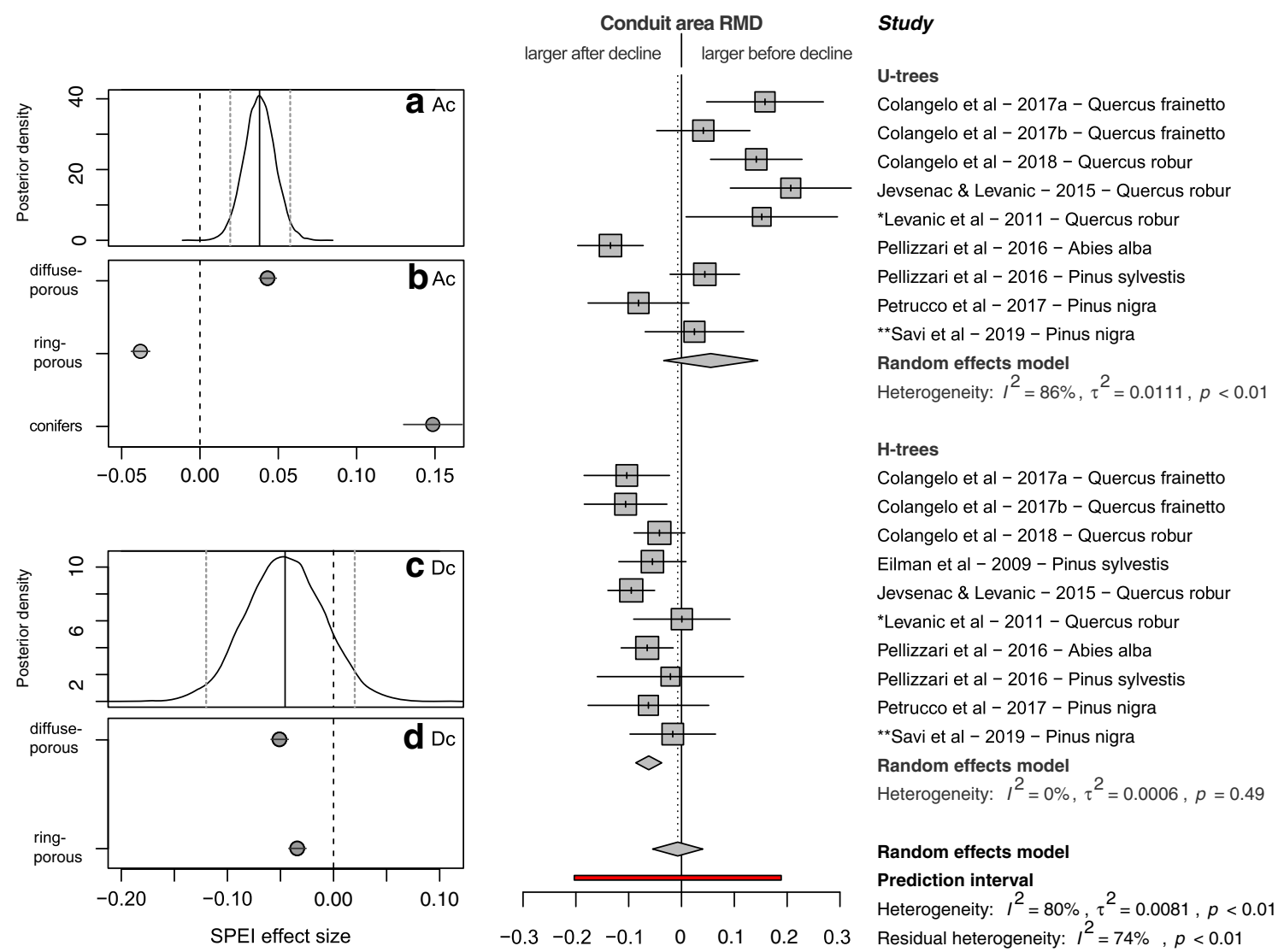

mean difference of samples and $\mathrm{x}$ is the sample mean) between xylem conduit area values measured before and after the onset of droughtinduced decline for H-trees and U-trees from selected study cases. Gray squares indicate the RMD and bars are $95 \%$ CIs. $I^{2}$ is the variability percentage independent of sampling error; $\tau^{2}$ is the between-study variance. For each study, the weight is computed as the inverse of within-study squared standard error plus the between-study variance, $\tau^{2}$. Diamonds represent summary RMDs between the values measured before and after the onset of tree decline. The vertical dashed line represents the line of no effect and the red line shows the range in which we can expect the effects of future studies to fall based on the current meta-analysis. *, hydraulic diameter; **, radial lumen diameter 

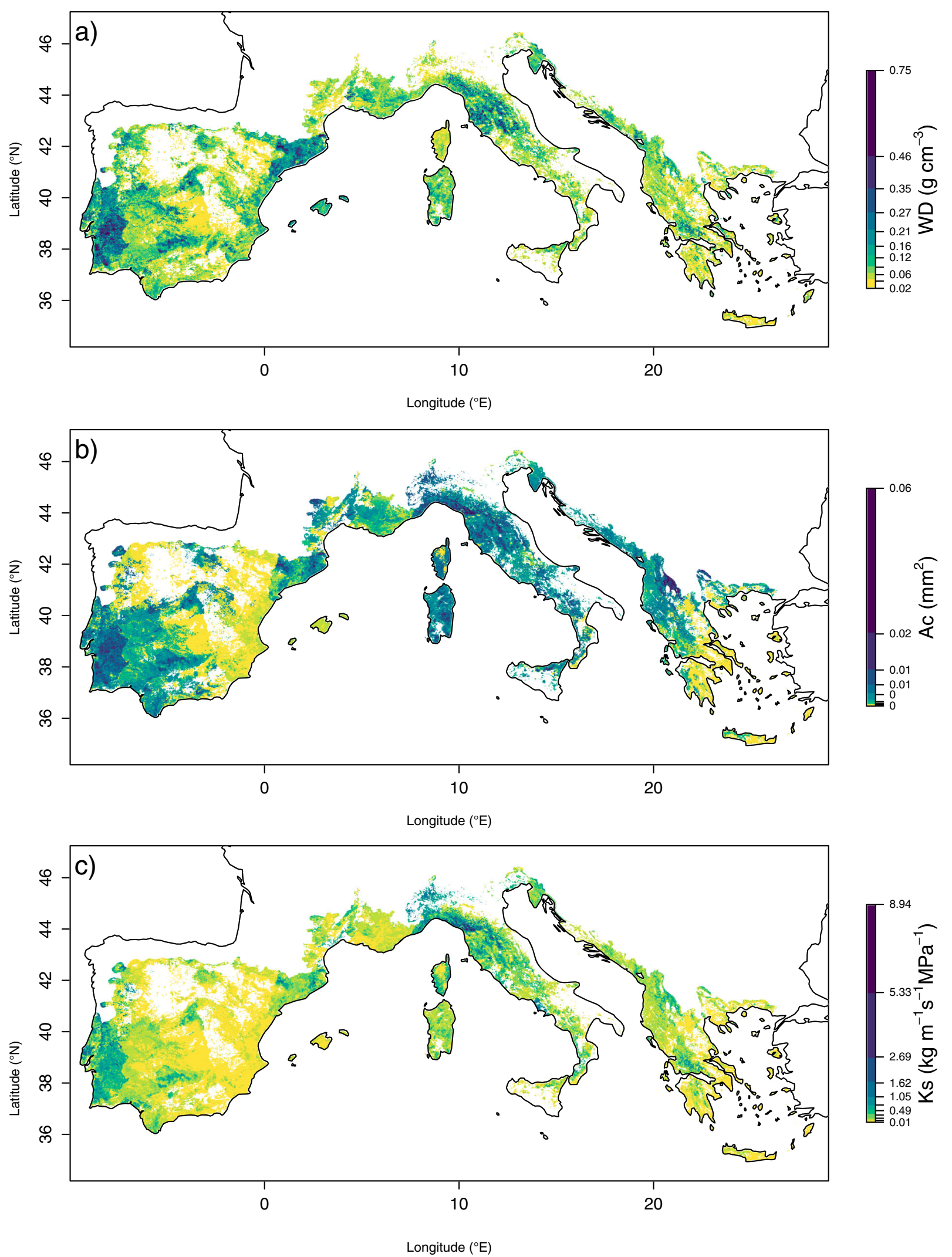

Fig. 5 Geographic maps of wood density (WD, upper panel), xylem conduit area (Ac, middle panel), and hydraulic specific conductivity (Ks, lower panel) for the Mediterranean region. The pixel level values were weighted on the relative abundance of each species/genus in the pixel. WD values of Castanea spp., Eucalyptus spp., Pseudotsuga spp., and Robinia spp. are from extra Mediterranean areas. Missing Ks values of Quercus robur/petraea were filled with the average Quercus spp. values 